\title{
Optimization of the Epitaxial Design of High Current Density Resonant Tunneling Diodes for Terahertz Emitters
}

\author{
Razvan Baba *a , Benjamin J. Stevens ${ }^{\mathrm{b}}$, Toshikazu Mukaic, Richard A. Hogg ${ }^{\mathrm{a}}$ \\ ${ }^{a}$ School of Engineering, University of Glasgow, Glasgow, G12 8LT, UK, \\ ${ }^{b}$ Dept. of Electronic \& Electrical Engineering, Centre for Nanoscience \& Technology, University of \\ Sheffield, Sheffield, S3 7HQ, UK \\ ${ }^{\mathrm{c}}$ Rohm Co. Ltd., Ukyo-ku, Kyoto 615-8585, Japan
}

\begin{abstract}
We discuss the numerical simulation of high current density InGaAs/AlAs/InP resonant tunneling diodes with a view to their optimization for application as $\mathrm{THz}$ emitters. We introduce a figure of merit based upon the ratio of maximum extractable $\mathrm{THz}$ power and the electrical power developed in the chip. The aim being to develop high efficiency emitters as output power is presently limited by catastrophic failure. A description of the interplay of key parameters follows, with constraints on strained layer epitaxy introduced. We propose an optimized structure utilizing thin barriers paired with a comparatively wide quantum well that satisfies strained layer epitaxy constraints.
\end{abstract}

Keywords: resonant tunneling diode, terahertz emitter, epitaxial optimization, RTD growth, misfit strain

\section{INTRODUCTION}

The ever increasing connectivity of the world is driving the need for wireless communication technologies, with the $\mathrm{THz}$ region attracting attention due to the possibility of high data rates with advantages in enhanced security and reduced network interference. Resonant tunneling diodes (RTDs) are high speed oscillators which have been coupled to antennae to realize effective room temperature emitters of $\mathrm{THz}$ radiation, demonstrating $1.92 \mathrm{THz}$ through harmonics ${ }^{1}$. The InGaAs/AlAs/InP material system is advantageous in terms of high electron mobility, suitable band-offsets, and offers low resistance contacts.

In this paper we explore the design of high performance RTDs using a 1D non-equilibrium Green's function simulator ${ }^{2}$. For this purpose, we propose a figure of merit related to the extraction efficiency of the radiated submillimetre wave emission power (Fig. 1). Previous studies have proposed using the peak current density, peak to valley current ratio $(\mathrm{PVCR})^{3}$, resonance linewidth ${ }^{4}$ or a combination thereof, but we consider that these are not the ideal quantities when attempting to maximise the $\mathrm{THz}$ emission power from an RTD, as these are measures of the internal quantum efficiency of the device.

\section{RTD MODELLING}

\subsection{Modelling software and method}

The modelling package ${ }^{2}$ approximates the solutions of the Schrödinger equation in a 1D crystal growth direction, taking into account the Fermi and Poisson distributions, effectively becoming a multi-body electron-electron interaction calculator. This is achieved via the non-equilibrium Green's functions(NEGF) method ${ }^{5}$, which has become the method of choice for a full quantum transport treatment of devices where incoherent scattering plays an important role ${ }^{6-10}$. The transmission matrix method ${ }^{11}$ is then implemented for every applied bias to determine the quasi-bound well levels. We may note that whilst the structure belongs to the superlattice category, the resulting carrier resonance formula in a double-barrier intraband RTD is mathematically equivalent to a Fabry-Perot etalon ${ }^{12}$. Despite the computational complexity, several works from different decades have used the NEGF method with at least good qualitative correlation to experimental measurements ${ }^{13-16}$.

The simulation does not fully accommodate the complex transport modes under thermally activated tunnelling and rough material interfaces. In this regard, the density matrix method has been proposed to overcome these limitations ${ }^{17}$, however, we achieved a similar goal empirically matching the scattering potential (a quantity in the Green's function 
introduced to take into account wave-packet perturbation). With this fitting parameter alone, it is possible to obtain a current density estimation within less than an order of magnitude. In addition to epitaxial growth errors, the departure from reality may arise from peripheral current leakage, deep trap levels, contact resistance, all which hint towards a large dependence on the fabrication process and material quality on the final experimentally obtained V-I, as evidenced in ${ }^{18}$. However, we have found that a 2-band $k \cdot p$ model is sufficient in most numerical simulation of our Type I quantum well (QW) RTD structures. The model successfully simulates the intrinsic hysteresis of structures described in the literature $^{19}$, a point touched on in Section 3 of this work.

Another limitation of this work is that NEGF simulators cannot predict the line-loaded case of a fabricated RTD, including an external resonator or antenna elements and contributions from contact resistance. The resulting I-V characteristic, particularly pertaining to the valley current would be different, though there are measurement techniques that involve less current averaging and employ the use of radiation absorber materials ${ }^{20}$, practically minimizing the difference.

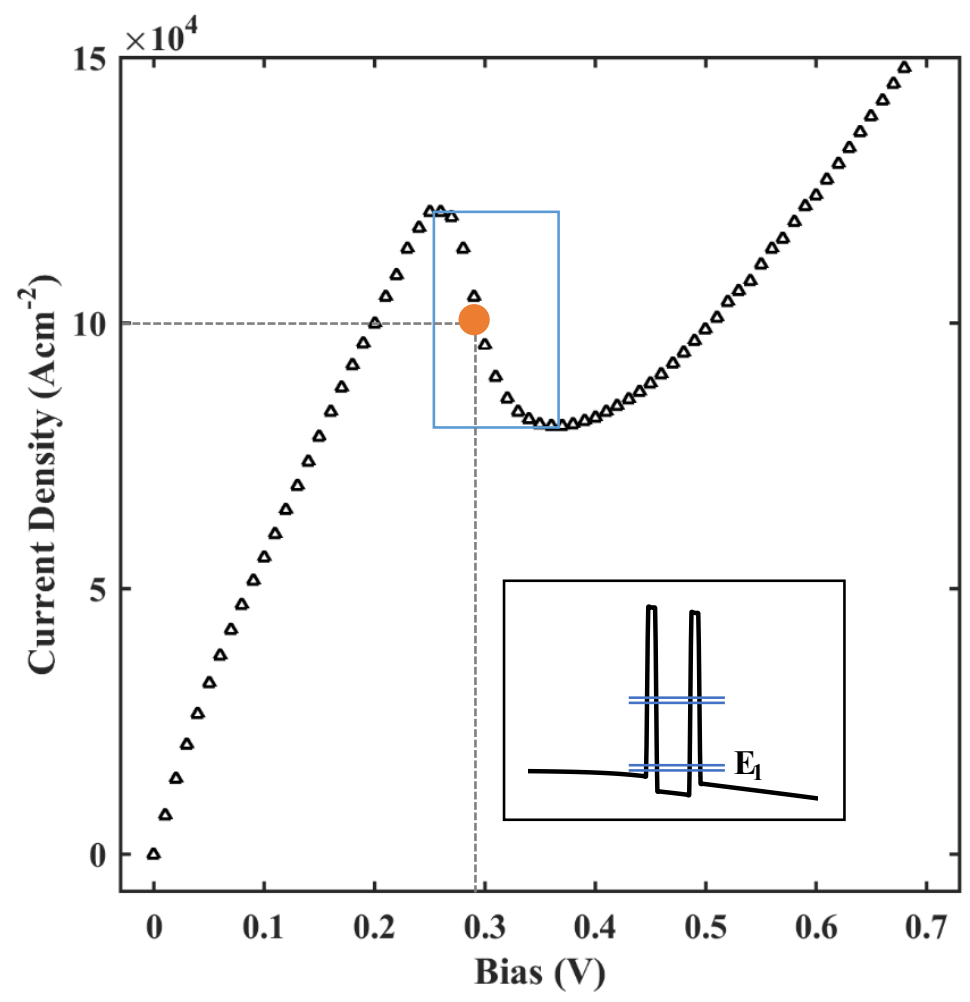

Figure 1. Modelled RTD I-V characteristic shown with electrical chip power (mid-point of negative differential conductance) and extractable power. (inset) Conduction band profile under bias near resonance, shown with 2 quasibound states.

\subsection{Modelled reference device}

The MOVPE growth of the InGaAs/AlAs RTD on an InP substrate is described in ${ }^{21}$. The modelled structure consists of i) $20 \mathrm{~nm} \mathrm{In} 0 .{ }_{0} \mathrm{Ga}_{47} \mathrm{As}, 3 \cdot 10 \mathrm{e} 18$ ii) 7 monolayer (MLs) $\mathrm{In}_{0.53} \mathrm{Ga}_{47} \mathrm{As}$ injector iii) $4 \mathrm{MLs} \mathrm{AlAs}$ iv) $15 \mathrm{MLs}^{\mathrm{In}} \mathrm{In}_{0.8} \mathrm{Ga}_{0.2} \mathrm{As}$ (QW) v) 4MLs AlAs vi) $20 \mathrm{~nm} \mathrm{In} 0 . \mathrm{I}_{3} \mathrm{Ga}_{47} \mathrm{As}$ collector vii) $25 \mathrm{~nm} \mathrm{In}_{0.53} \mathrm{Ga}_{.47} \mathrm{As}, 3 \cdot 10 \mathrm{e} 18$. This reference structure has been used to yield a peak current density of $\sim 700 \mathrm{kAcm}^{-2}$, used to generate a primary oscillation of $353 \mathrm{GHz}$ when coupled into a slot antenna ${ }^{22}$. Figure 1 shows representative I-V characteristic of these devices ${ }^{22}$, together with the modelled fit which provides the baseline for this paper. Full Hartree potential calculations are employed in the area including the $2 \mathrm{~nm}$ entry spacer, barriers, and quantum well (QW) layers. The rest of the model uses the bulk approximation to save computational time with minimal loss of fidelity. 


\section{NATURE OF RTD RESONANCE}

One of the main issues in estimating the oscillator output power points back to the reported hysteretic region of measured $\mathrm{I}-\mathrm{V}$ curves $^{23,24}$. However, the nature of an observed hysteresis can be either due to the intrinsic bistability of the device ${ }^{19}$, ${ }^{25}$, i.e quantum well charge accumulation and depletion due to quasi-bound level mis-alignment, or extrinsic, due to the external circuit and parasitic components such as contact resistance, inductance and line-loading effects ${ }^{26}$. An extensive modelling study has also been made to ascertain the effect of various design parameters on the hysteresis and plateau region $^{27}$ for memory applications.

We therefore investigated the nature of the intrinsic oscillation, and whether this comparatively slow charge-build-up and release is an issue in our structures. In this regard, we simulated an asymmetric barrier GaAs/AlGaAs device which has been shown to exhibit strong intrinsic bistability ${ }^{19}$. Figure 2 plots our simulation results (ascending and descending voltage sweeps) in addition to experimental data extracted from ${ }^{19}$ at $4 \mathrm{~K}$. The time averaged experimental data can be well explained by the superposition of the ascending and descending sweep simulations indicating how the simulator can be utilized to analyze the relative importance of intrinsic oscillation in RTDs. Simulations for our high current density structures are plotted in Figure 3, which show limited difference between forward and reverse sweeps. The small differences are attributed to the difference in the undoped spacer layers of the structure. We confirm that oscillation is therefore dominated by the higher frequency extrinsic bistability in our high current density RTD structures.

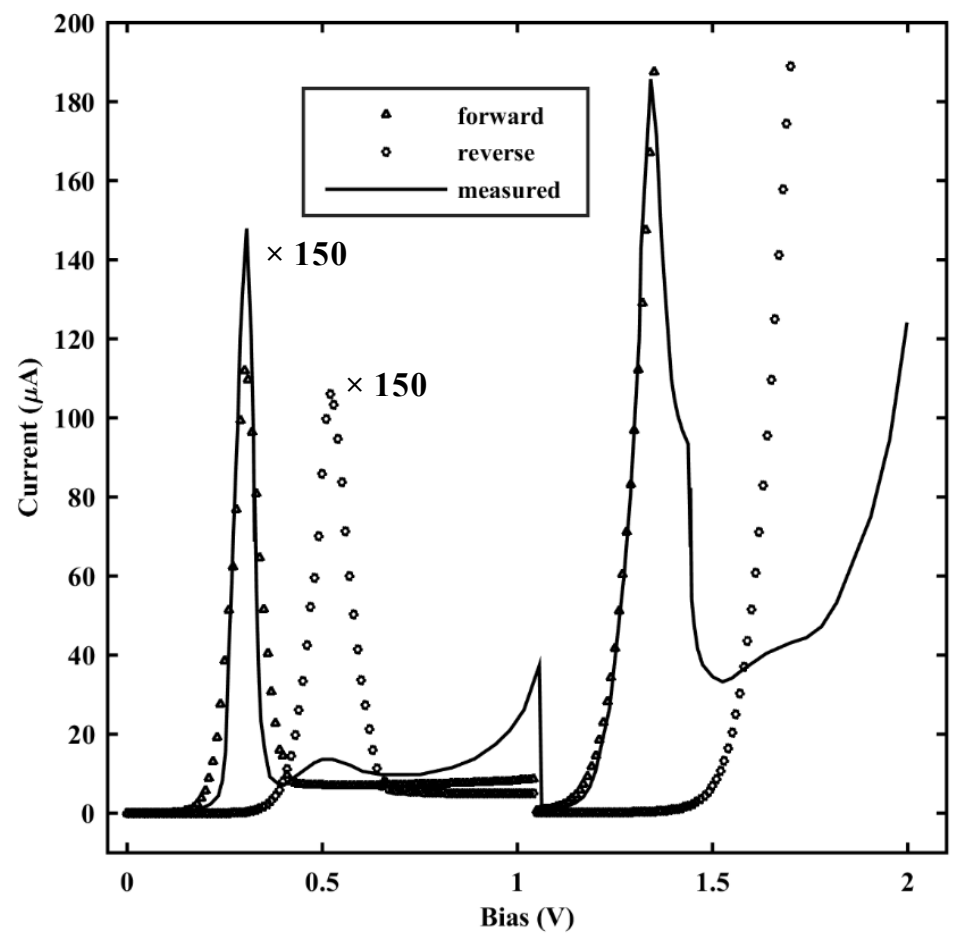

Figure 2. Modelled forward and reverse voltage sweeps of a GaAs-based RTD at 4K fit to data from [6], showing the intrinsic bistability of the device 


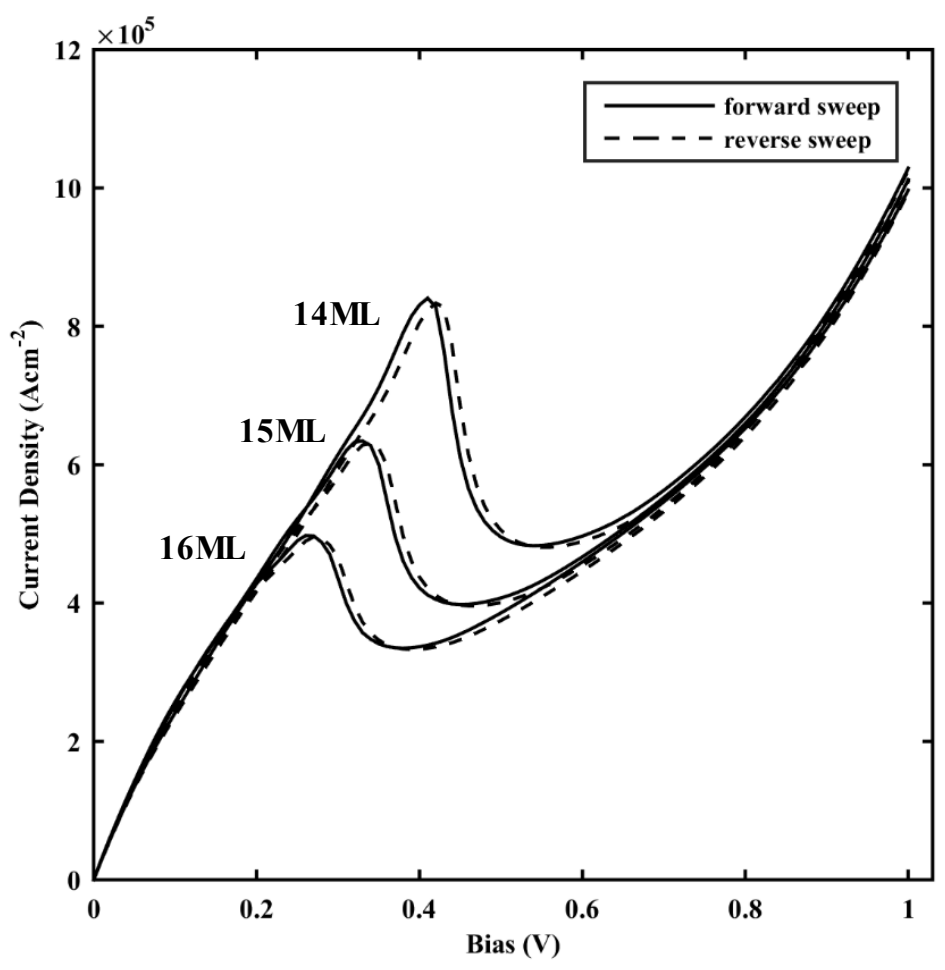

Figure 3 Modelled forward and reverse voltage sweeps of our reference RTD design, with different QW widths.

\section{FIGURE OF MERIT}

Our figure of merit is composed of two measures: the time-averaged electrical chip power, $\mathrm{P}_{\text {chip }}$, and the extractable THz power, $\mathrm{P}_{\mathrm{THz}}$, that is derived from the 3rd order polynomial approximation of the I-V plot, which can be re-written as a Van der Pol oscillator characteristic ${ }^{28}$ The chip power is given by

$$
\mathrm{P}_{\text {chip }}=\mathrm{I} \mathrm{V}
$$

Where the bias point is chosen as the mid-point of the negative differential conductance (NDC), as seen in Figure 1, as measurements have shown this to be a point of maximum THz emission ${ }^{29}$. The extractable $\mathrm{THz}$ power is defined as ${ }^{28}$

$$
\mathrm{P}_{\mathrm{THz}}=3 / 16 \Delta \mathrm{I} \Delta \mathrm{V}
$$

Where $\Delta \mathrm{I}$ and $\Delta \mathrm{V}$ are defined by the peak and valley currents and voltages, respectively. These values are highlighted schematically in Fig. 1. We therefore define our figure of merit (FOM) as

$$
\mathrm{FOM}=\mathrm{P}_{\mathrm{THz}} / \mathrm{P}_{\text {chip }}
$$

Since $\mathrm{P}_{\text {chip }}>\mathrm{P}_{\mathrm{THz}}$, the ratio will be always subunitary and is a measure of the radiative extraction efficiency of the RTD.

\section{RESULTS AND DISCUSSION}

Our investigation proceeds with varying the 3 key parameters of the RTD: the barrier width, QW width, and QW depth. Firstly, we present the data without any regard to lattice misfit strain, then this consideration is added to the analysis to suggest the best possible epitaxial structure for a $\mathrm{THz}$ emitter. In order to give an unambiguous calculation, all dimensions are expressed in monolayer sheets $(M L s)$. One sheet is estimated at a half-distance of the InP substrate lattice constant, $\sim 0.293 \mathrm{~nm}$. 


\subsection{Alteration of principal device design parameters}

Figure 4 plots the FOM for our standard structure varying (a) the AlAs barrier width, (b) the QW width in ML, and (c) the QW indium composition. The same vertical scale is used for ease of comparison. Changing the barrier width (Fig 4(a) produces effects in line with previous publications ${ }^{3}$, in that there is an exponential increase in peak current at the expense of PVCR; In most structures, there is an optimum occurring at around 3MLs. However, our figure of merit is maximised when the barriers are set to $2 \mathrm{MLs}$ thickness, due to the change in the NDC, something which conventional figures of merit neglect to take into account. At $1 \mathrm{ML}$ barrierss, the loss of confinement is pronounced as the non-linear behaviour is subdued, whereas our model could not predict the resonance at 6MLs, which can be attributed to the lack of taking into account the changing strain conditions $\mathrm{s}^{30}$, as well as an expected change in the main fitting parameter. We mention that the maximum peak current density recorded in this case would reach values of $2.5 \mathrm{MAcm}^{-2}$, requiring a device diameter $<1 \mu \mathrm{m}$ and additional consideration for heat dissipation.

Increasing the QW width (Figure 4(b)) brings the quasi-bound resonant levels closer to the conduction band potential limit, at the expense of an increased carrier dwell time ${ }^{20}$, in effect trading off high power output with the cut-off frequency. Our reference structure with 4MLs barrier reaches an optimum with a 13MLs QW.

Increasing the well depth is achieved by altering the indium composition in the InGaAs alloy. This has a two-fold effect in that the incorporation of further indium not only alters the band gap, but the lowered effective mass translates into a decrease in the available density of states, resulting in a lower peak current density. This however, also reduces the operational bias of the device, which in practice, would lead to an expected increase in operational life time and reduced phase noise. With that in mind, in most of our simulations, the addition of extra indium up to InAs has no negative effect (Figure $4 \mathrm{c}$ ), unless $\mathrm{E}_{1}$ falls below the conduction band, which happens with a particular combination of thin well/high indium concentration.

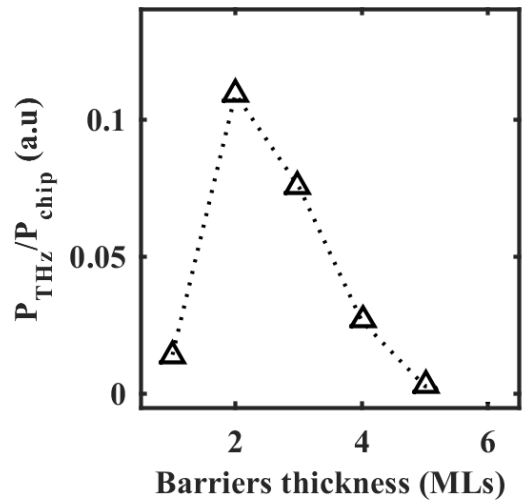

(a)

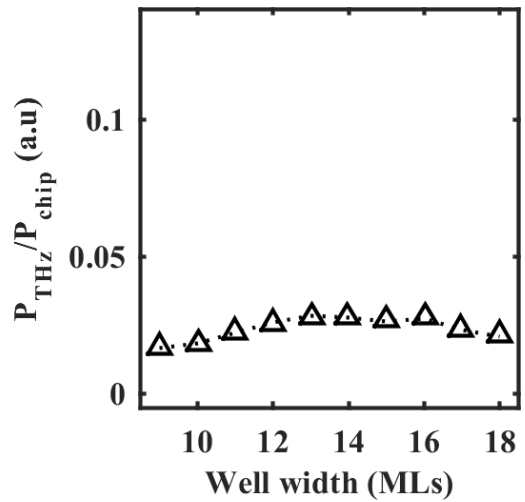

(b)

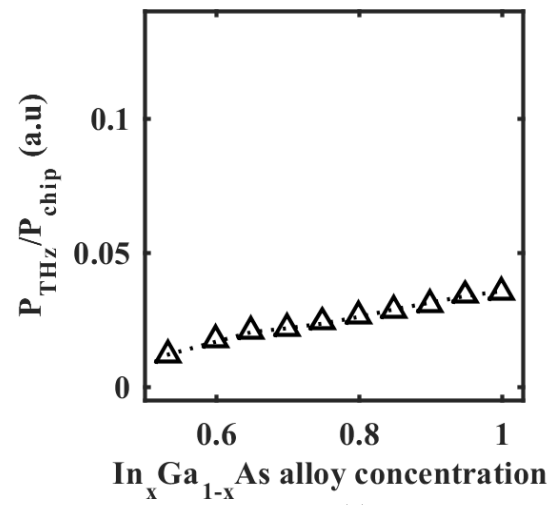

(c)

Figure. 4 The variation of our figure of merit shown against individual changes from the reference structure: (a) the width of the barriers in monolayers, (b) the QW width in monolayers (c) varying the depth of the QW through the composition of the ternary alloy

\subsection{Strain considerations and manufacturability}

The effect of strain has been intentionally ignored for the previous analysis. The coherent growth of strained layers upon a substrate without the formation of dislocations is a key challenge for epitaxy.

There are several models available to calculate the so-called critical thickness for a layer of given strain. One of the most referenced works in the field by Matthews \& Blackeslee ${ }^{31}$ is based purely on mechanical equilibrium theory, and yields a lower theoretical limit of the critical strain thickness. This does not take into account the complexities of ternary alloys, as it was indeed conceived with nearly lattice-matched compounds. Instead, People \& Bean ${ }^{32,33}$ have done direct additions to the work, adding a quadratic spline that would better fit the realities of heavily strained structures.

The growth of strained layers, starin-balanced QWs and our partially strain balanced structures is shown schematically in Fig 5. Figure 5(a) shows the case for the growth of a strained layer upon unstrained layers. In this case stress accumulates in the structure until a force $(\mathrm{M} \& \mathrm{~B})$ or energy $(\mathrm{P} \& \mathrm{~B})$ limit is reached (shown by a dotted line) and misfit 
dislocations are formed. Figure 5(b) shows the case for so-called "strain-balanced" structures ${ }^{34}$ where the average lattice constant of the structure is designed to be equal to that of the substrate in order to overcome limitations in growing multiple QW layers as in Fig 5(a). This situation actually balances accumulated stress within the structure. Our situation is shown in Fig 5(c) where partial strain-balancing is achieved, with an InGaAs QW (+1.72\% lattice constant) and binary AlAs barriers (-5.32\% lattice constant).

Of the parameters used in the computation to adjust to InGaAs/AlAs on InP would be the lattice constant taken from ${ }^{35}$, Poisson ratio from ${ }^{36}$ (assuming linear interpolation for InGaAs between GaAs and InAs) and a Burgers' vector assumed at $4 \AA$ as per the corrected $P \& B^{33}$ model. In equation (1) of the same paper, the coefficient for $\operatorname{In}_{0.8} \mathrm{Ga}_{0.2} \mathrm{As}$ is $1.855 \cdot 10^{-2} \AA$ and for AlAs $2.094 \cdot 10^{-2} \AA$.

Figure 6 plots the FOM as a function of well width and indium composition for an RTD design having 3ML barrier layers. Thick lines are added for structures which satisfy the requirements for misfit dislocation formation after People $\&$ Bean $^{32}$. From analysis of this graph, we suggest an optimal device structure with 3MLs barriers, 14ML QW and a QW alloy of $\operatorname{In}_{0.9} \mathrm{Ga}_{0.1}$ As.

The calculated I-V of the optimised structure is plotted in Figure 7. It exhibits similar biasing requirements, higher PVCR, and $\times 4$ increase in peak current compared to the reference design. We note again that this structure is not strain balanced, but designed to be compressively strained to the maximum acceptable limit based on the adjusted projection of the P\&B model, in order to avoid the onset of the generation of misfit dislocations. We also note that our findings indicate the challenge now made to the epitaxial process in realizing high performance $\mathrm{THz}$ emitters. Not only are highly uniform thin layers required (e.g. 2ML AlAs barriers), but the limits of strain relaxation must be pushed in order to maximise efficiency of these THz light sources.

\section{CONCLUSION}

We have described a novel figure of merit based on measurements from our own fabricated RTDs that can be used as a design aid to maximize $\mathrm{THz}$ radiation output. We highlight the importance of the chip power and explain how the variation of certain epitaxial parameters affects the efficiency of the device. An optimal device is proposed by varying 3 essential geometrical parameters. The practicality of manufacturing this device has been discussed in terms of strain relaxation and the formation of misfit dislocations. 


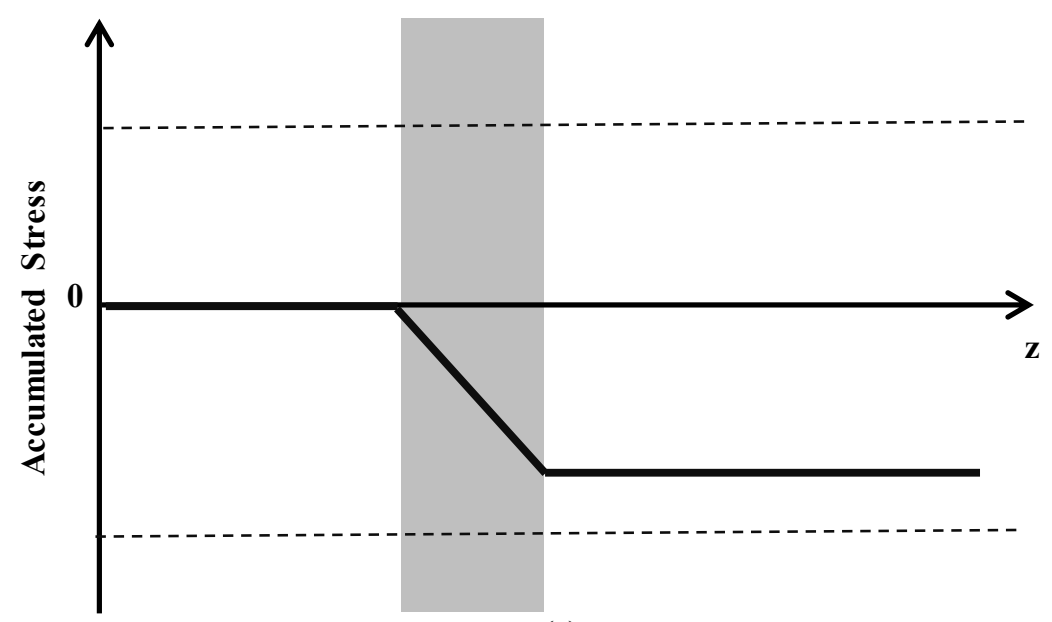

(a)

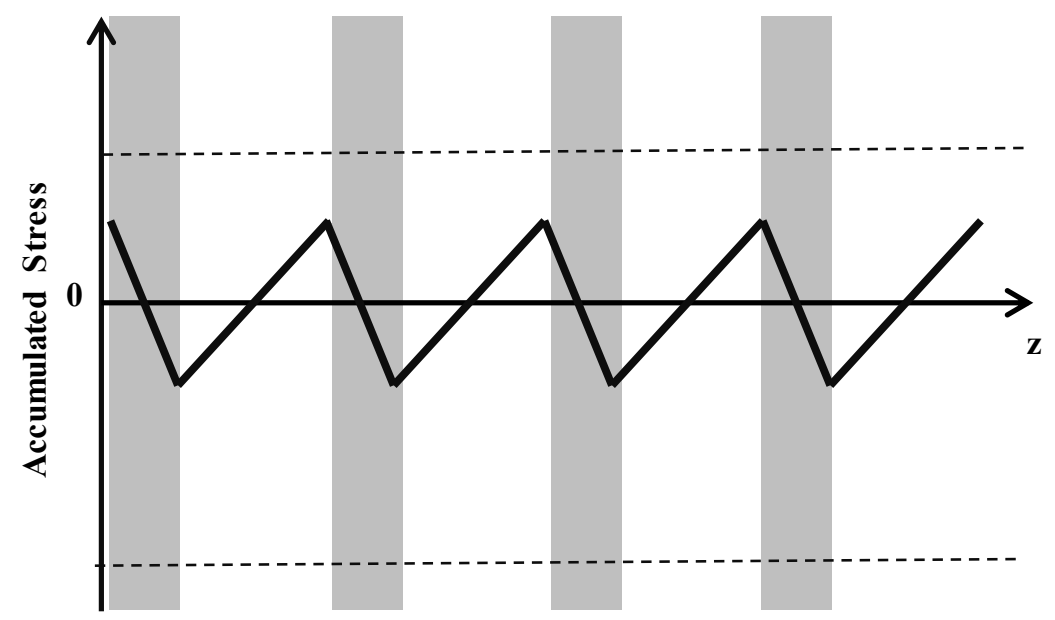

(b)

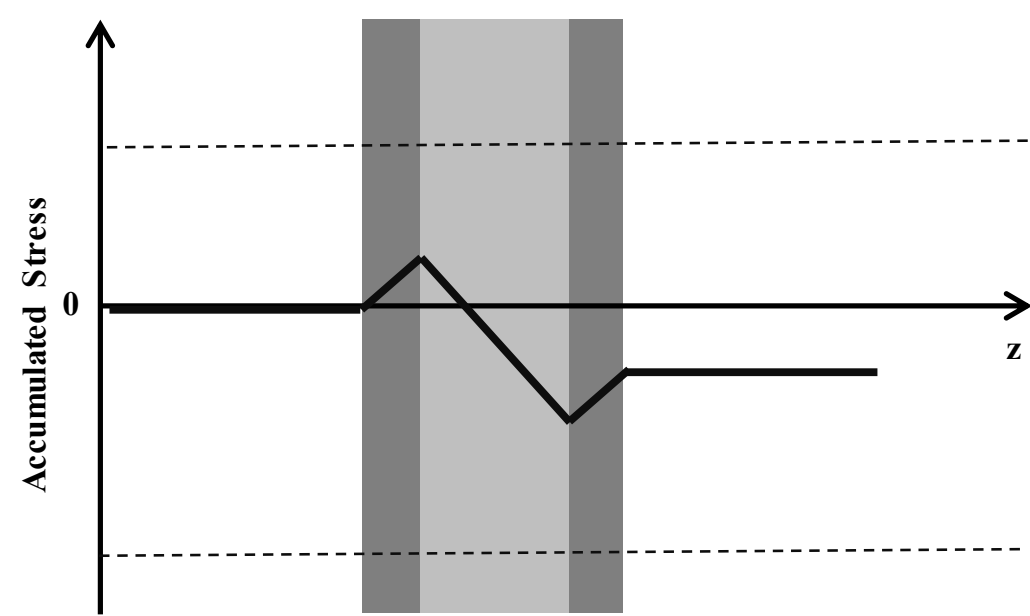

(c)

Figure 5. Schematic of (a) strained layer grown within an un-strained matrix. (b) strain-balancing in an MQW stack incorporating alternating compressive and tensile strained layers, and (c) the situation for our structures which incorporates partial strain-balancing. 


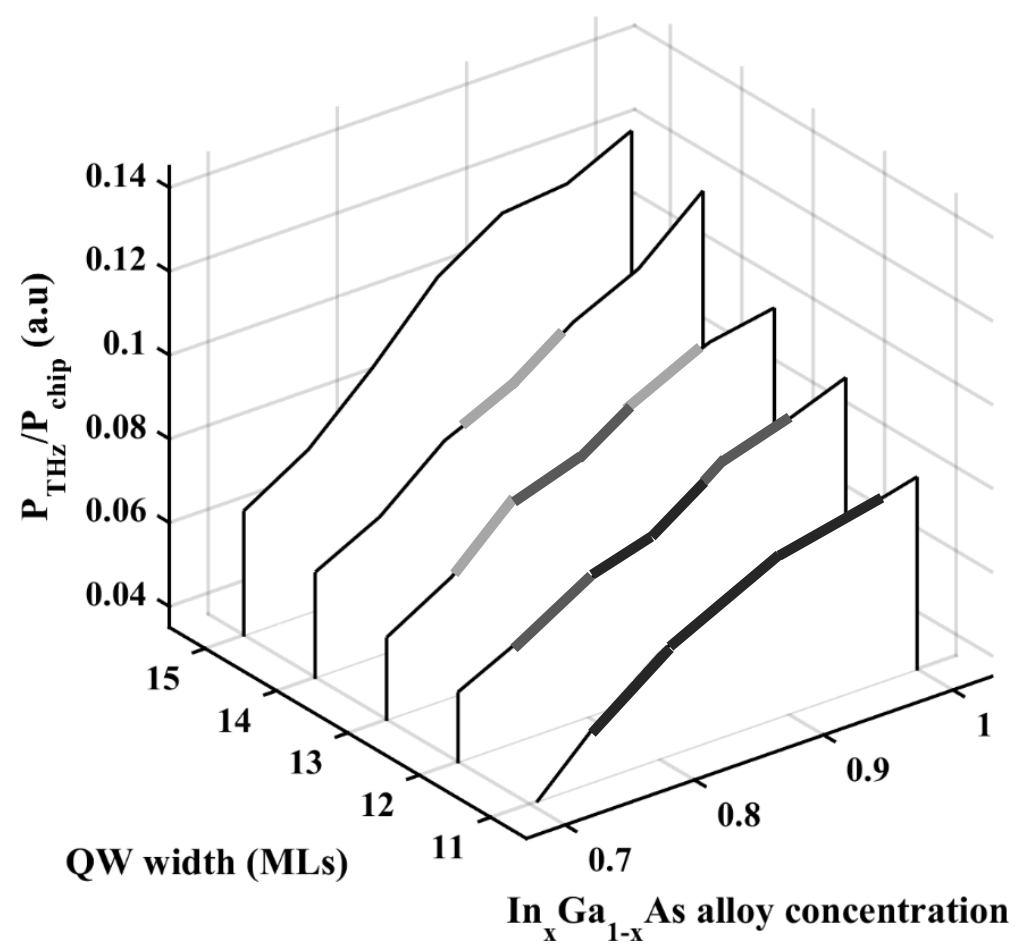

Figure 6. The variation in the figure of merit using 3MLs AlAs barriers. The gray level overlays indicate the strain on the structure to be grown, with lighter levels showing a lesser confidence on manufacturability

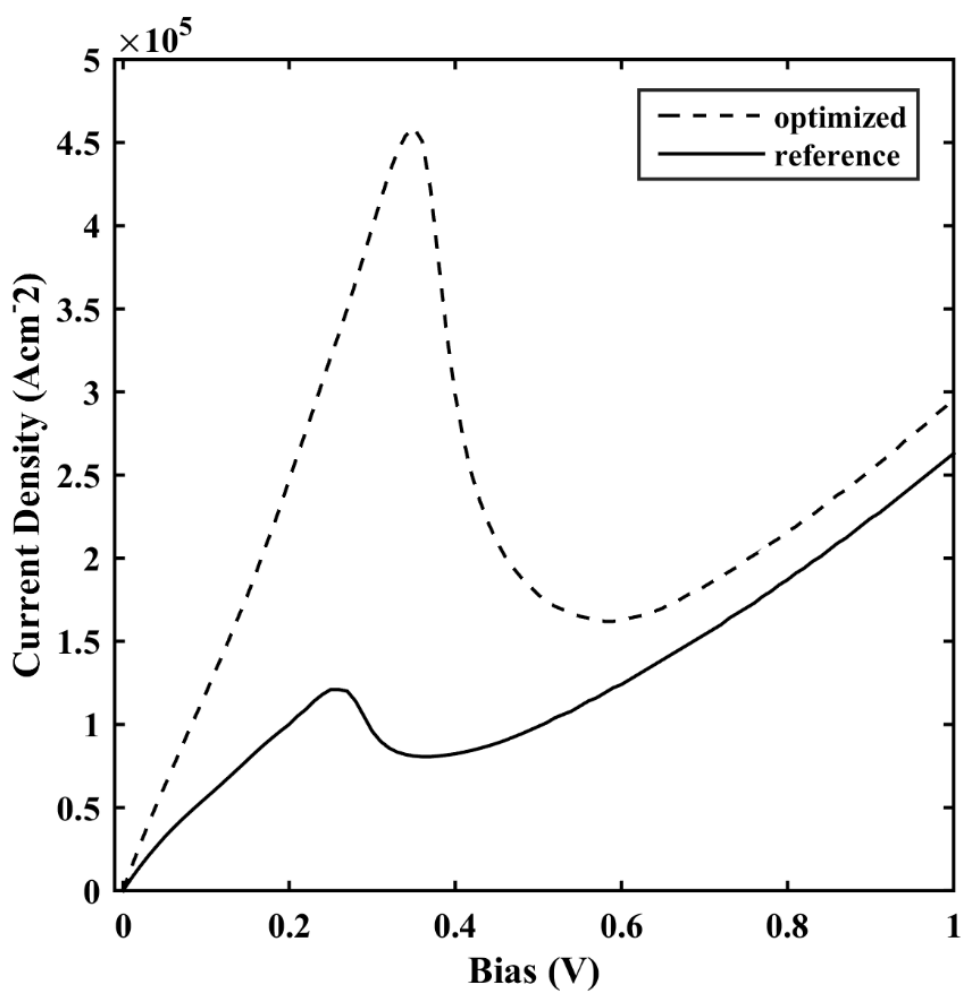

Figure 7. Comparative I-V between current and proposed device 


\section{REFERENCES}

[1] Maekawa, T., Kanaya, H., Asada, M.., Suzuki, S., “1.92 THz Oscillator using Resonant Tunneling Diode Integrated with Slot Antenna with Reduced Conduction Loss," 3rd Int. Symp. Microwave/Terahertz Sci. Appl. (MTSA 2015), Okinawa (2015).

[2] Indlekofer, K. M.., Malindretos J., “'WinGreen' - Simulation of semiconductor nanodevices,” 2004, $<$ http://www.hs-rm.de/ing/ueber-uns/personen/personen-im-fb-ing/prof-dr-klaus-michael-indlekofer/forschungund-entwicklung/nanoelektronik/>.

[3] Sugiyama, H., Teranishi, A., Suzuki, S.., Asada, M., "Structural and electrical transport properties of MOVPEgrown pseudomorphic AlAs/InGaAs/InAs resonant tunneling diodes on InP substrates,” Jpn. J. Appl. Phys. 53(3), 31202 (2014).

[4] Zainal, N., Walker, P.., Kent, A. J., "Modelling of cubic AlxGa1-xN/GaN resonant tunnel diode structures," Phys. status solidi 7(7-8), 2262-2264, WILEY-VCH Verlag (2010).

[5] Jauho, A., "Introduction to the Keldysh nonequilibrium Green function technique," Lect. notes, 17 (2006).

[6] Vogl, P.., Kubis, T., “The non-equilibrium Green's function method: An introduction,” J. Comput. Electron. 9, 237-242 (2010).

[7] Strianti, G., "Application of the Green's Functions Method to the Study of the Optical Properties of Semiconductors," Riv. del Nuovo Cim. 11(12), 1 (1988).

[8] Birner, S.., Kubis, T., "Ballistic current in a resonant tunneling diode (RTD)," 2014, $<$ http://www.nextnano.com/nextnano3/tutorial/1Dtutorial_RTD_green.htm> (7 October 2014 ).

[9] Sandu, T.., Kirk, W. P., "The role of emitter quasi-bound state and scattering on intrinsic bistability in resonant tunneling diodes," Phys. E Low-Dimensional Syst. Nanostructures 22(4), 815-824 (2004).

[10] Datta, S.., van Houten, H., "Electronic Transport in Mesoscopic Systems," Phys. Today 49, 70 (1996).

[11] Tsu, R.., Esaki, L., "Tunneling in a finite superlattice," Appl. Phys. Lett. 22(11), 562-564 (1973).

[12] Moskalyuk, V.., Fediai, A., "Simplified analytical model of resonant-tunneling diode," Electron. Technol. 2009. ISSE 2009. 32nd Int. Spring Semin., 1-5 (2009).

[13] Söderström, J. R., Brown, E. R., Parker, C. D., Mahoney, L. J., Yao, J. Y., Andersson, T. G.., McGill, T. C., "Growth and characterization of high current density, high-speed InAs/AlSb resonant tunneling diodes," Appl. Phys. Lett. 58(3), 275 (1991).

[14] Schulman, J. N., De Los Santos, H. J.., Chow, D. H., "Physics-based RTD current-voltage equation," Electron Device Lett. IEEE 17(5), 220-222 (1996).

[15] Voves, J., Třebický, T.., Jackiv, R., "NEGF simulation of the RTD bistability," J. Comput. Electron. 6(1-3), 259-262 (2007).

[16] Feiginov, M., Sydlo, C., Cojocari, O.., Meissner, P., "Resonant-tunnelling-diode oscillators operating at frequencies above 1.1 THz," Appl. Phys. Lett. 99(23), 233506 (2011).

[17] Terazzi, R.., Faist, J., “A density matrix model of transport and radiation in quantum cascade lasers,” New J. Phys. 12(3), 33045 (2010).

[18] Jacobs, K. J. P., Stevens, B. J., Wada, O., Mukai, T., Ohnishi, D.., Hogg, R. A., “A dual-pass high current density resonant tunnelling diode terahertz emitter," Infrared, Millimeter, Terahertz waves (IRMMW-THz), 2015 40th Int. Conf., 1-2 (2015).

[19] Pate, M. A., “Observation of intrinsic bistability in resonant tunnelling devices," Electron. Lett. 24(18), 11901191(1), Institution of Engineering and Technology (1988).

[20] Feiginov, M., Sydlo, C., Cojocari, O.., Meissner, P., "High-frequency nonlinear characteristics of resonanttunnelling diodes," Appl. Phys. Lett. 99 (2011).

[21] Jacobs, K. J. P., Stevens, B. J., Mukai, T., Ohnishi, D.., Hogg, R. A., "Non-destructive mapping of doping and structural composition of MOVPE-grown high current density resonant tunnelling diodes through photoluminescence spectroscopy," J. Cryst. Growth 418, 102-110, Sheffield (2015).

[22] Jacobs, K. J. P., Stevens, B. J., Wada, O., Mukai, T., Ohnishi, D.., Hogg, R. A., “A Dual-Pass High Current Density Resonant Tunneling Diode for Terahertz Wave Applications," Electron Device Lett. IEEE 36(12), 1295-1298 (2015).

[23] Goldman, V. J., Tsui, D. C.., Cunningham, J. E., "Observation of intrinsic bistability in resonant tunneling structures,” Phys. Rev. Lett. 58(12), 1256-1259, American Physical Society (1987).

[24] Sollner, T. C. L. G., "Comment on observation of intrinsic bistability in resonant-tunneling structures," Phys. Rev. Lett. 59(14), 1622 (1987). 
[25] Sandu, T.., Kirk, W. P., "Intrinsic bistability and emitter scattering in resonant tunneling diodes," Phys. E LowDimensional Syst. Nanostructures 19(1-2), 83-88 (2003).

[26] Liu, H. C., "Simulation of extrinsic bistability of resonant tunneling structures," Appl. Phys. Lett. 53(6), 485 (1988).

[27] Zhao, P., Cui, H. L., Woolard, D., Jensen, K. L.., Buot, F. A., "Simulation of resonant tunneling structures: Origin of the I-V hysteresis and plateau-like structure," J. Appl. Phys. 87(3), 1337 (2000).

[28] Kim, C. S.., Brandli, A., "High-Frequency High-Power Operation of Tunnel Diodes," Circuit Theory, IRE Trans. 8(4), 416-425 (1961).

[29] Jacobs, K. J. P., Stevens, B. J., Wada, O., Mukai, T., Ohnishi, D.., Hogg, R. A., "Micron-Scale Resonant Tunnelling diodes for THz applications through standard I-line Photolitography," Sheffield (2015).

[30] Miyamoto, Y., Tobita, H., Oshima, K.., Furuya, K., "Barrier thickness dependence of peak current density in GaInAs/AlAs/InP resonant tunneling diodes by MOVPE," Solid. State. Electron. 43(8), 1395-1398 (1999).

[31] Matthews, J. W.., Blakeslee, A. E., “Defects in epitaxial multilayers,” J. Cryst. Growth 27, 118-125 (1974).

[32] People, R.., Bean, J. C., "Calculation of critical layer thickness versus lattice mismatch for GexSi1-x/Si strainedlayer heterostructures," Appl. Phys. Lett. 47(3), 322 (1985).

[33] People, R.., Bean, J. C., "Erratum: Calculation of critical layer thickness versus lattice mismatch for GexSi1-x/Si strained-layer heterostructures [Appl. Phys. Lett. 47, 322 (1985)],” Appl. Phys. Lett. 49(4), 229 (1986).

[34] Ekins-Daukes, N. J., Barnham, K. W. J., Connolly, J. P., Roberts, J. S., Clark, J. C., Hill, G.., Mazzer, M., "Strain-balanced GaAsP/InGaAs quantum well solar cells," Appl. Phys. Lett. 75(26), 4195 (1999).

[35] Vurgaftman, I., Meyer, J. R.., Ram-Mohan, L. R., "Band parameters for III-V compound semiconductors and their alloys,” J. Appl. Phys. 89, 5815-5875 (2001).

[36] Jain, S. C., Willander, M.., Maes, H., "Stresses and strains in epilayers, stripes and quantum structures of III - V compound semiconductors," Semicond. Sci. Technol. 11, 641-671 (1999). 